\title{
MÉTODOS DE ELETROMAGNETISMO PARA DETECÇÃO DE DESVIOS EMBUTIDOS DE ENERGIA ELÉTRICA
}

Felipe Curcino Nascimento ${ }^{1}$; Juliana Maria Souza de Santana²; Ane Caroline Teixeira da Paixão; Valmiro José Rangel Galvis²; Ricardo Cerqueira Medrado².

${ }^{1}$ SENAI CIMATEC - Área de Eletrônica Embarcada, Instituto SENAI de Inovação em Automação; Salvador/BA; felipecurcino@hotmail.com.br;

2 SENAI CIMATEC - Área de Eletrônica Embarcada, Instituto SENAI de Inovação em Automação; Salvador/BA.

Resumo: A detecção de desvios embutidos ainda é um problema para as concessionárias de energia elétrica, visto que os métodos utilizados não baseiam-se em tecnologias que garantam uma boa margem de assertividade. Além disso, a falta de comprovação do ilícito reforça a sensação de impunidade e torna a fraude recorrente, acarretando em constantes prejuízos para as concessionárias. Com base nisso, o presente artigo discorre acerca de métodos eletromagnéticos que podem ser empregados na detecção dos desvios embutidos visando um aumento no nível de assertividade das inspeções das equipes de combates ao furto de energia.

Palavras-Chave: Ondas Eletromagnéticas; Furto; Fraude; Detecção; Desvios.

\section{ELECTROMAGNETISM METHODS FOR BUILT-IN ELECTRICAL POWER THEFT DETECTION}

\begin{abstract}
The detection of built-in deviations is still a problem for electric power concessionaires, as the methods used are not based on technologies that guarantee a good assertiveness margin. In addition, the lack of proof of theft reinforces the sense of impunity and makes the fraud recurring, causing constant damage to the concessionaires. Based on this, the present article discusses about electromagnetic methods that can be employed in detecting built-in deviations aiming at an increase in the assertiveness level of inspections of combat teams to theft of energy.
\end{abstract}

Keywords: Electromagnetic waves; Theft; Fraud; Detection; Deviations. 


\section{INTRODUÇÃO}

Na contramão do desenvolvimento social e econômico do país, um ato delituoso tem causado grandes perdas financeiras às concessionárias de energia elétrica e aos consumidores: o furto de energia elétrica. Este prejuízo é classificado pela agência reguladora como perda não técnica, ou perda comercial, tendo em vista a Resolução Normativa 338/08 da ANEEL, onde a perda não técnica é o resultado da subtração da energia injetada no sistema (gerada), energia faturada (cobrada) e das perdas técnicas (perdas físicas) [1]. Dentre as principais origens das perdas não técnicas está desvio embutido. [2]

Esses desvios são comumente realizados através da criação de derivações em condutores antes do sistema de medição [3], possibilitando a utilização da energia elétrica da rede sem que seja contabilizada. Existem métodos empregados pelas concessionárias para a detecção destes desvios, no entanto, eles possuem como limitações: i) alto custo; ii) possível constrangimento ao consumidor; e, iii) a dificuldade de obtenção de provas do ilícito para responsabilização civil e criminal dos infratores.

O problema de pesquisa advém da necessidade de detectar desvios embutidos de maneira rápida, assertiva e com geração de provas do ilícito. Deste problema surge o questionamento: Quais métodos e tecnologias possibilitariam, com eficiência, a identificação dos desvios embutidos?

Visando solucionar o problema descrito, foi proposto pelo projeto de pesquisa (PD-0382-0121/2018):

- Estudo de métodos eletromagnéticos para detecção de objetos embutidos;

- Imageamento dos objetos identificados.

O presente artigo visa abordar possíveis métodos que podem ser utilizados para identificar fraudes embutidas em paredes para que, com técnicas menos invasivas, as concessionárias possam aumentar o nível de assertividade das operações.

Este trabalho foi desenvolvido no âmbito do Programa de Pesquisa e Desenvolvimento Tecnológico do Setor de Energia Elétrica regulado pela ANEEL e as reflexões e resultados apresentados são fruto do projeto de Pesquisa e Desenvolvimento "Identificação de fraudes embutidas por sobreposição de camadas de imagens". Código ANEEL PD-0382-0121/2018, em execução pelo Senai CIMATEC e com conclusão prevista para agosto/2020, realizado com suporte financeiro da Light Serviços de Eletricidade.

\section{METODOLOGIA}

As etapas iniciais do projeto dedicaram-se a uma revisão bibliográfica com estudo do princípio de funcionamento de diversos métodos para detecção de objetos através de paredes e como estes poderiam ser implementados para aprimorar as técnicas de combate ao furto de energia. Estas pesquisas foram feitas no SENAI CIMATEC e tiveram duração de 3 meses. 
Concluída a etapa informacional do projeto, as tecnologias identificadas foram comparadas quanto a sua capacidade de detectar desvios embutidos [4]. O período destinado para a avaliação das tecnologias e dados para compor a aplicação tiveram duração de 3 meses. A Figura 1 detalha as etapas envolvidas na produção do presente trabalho.

Figura 1. Etapas Metodológicas.

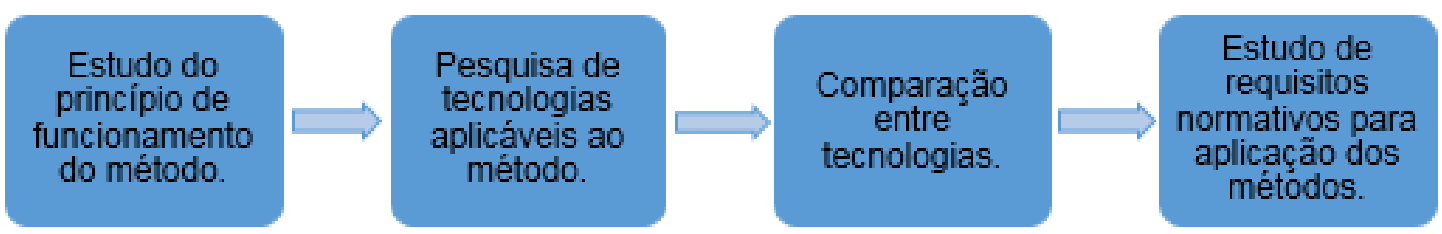

Fonte: Própria, 2019.

Conforme destacado na Figura 1, as tecnologias declaradas como viáveis foram comparadas, para que, a partir de então, fosse possível analisar quais métodos seriam úteis ou não para o problema de pesquisa. Da mesma forma, os requisitos normativos necessários para utilização dessas técnicas foram avaliados e levantados a partir de pesquisas bibliográficas. Todos os processos de pesquisa foram realizados na instituição SENAI CIMATEC.

\section{RESULTADOS E DISCUSSÃO}

\subsection{Técnicas de detecção de materiais através de paredes}

A técnica mais antiga utilizada para detecção de objetos através de paredes utilizava um sensor capacitivo que, ao fazer o mapeamento na área em que desejavase inspecionar, identificava objetos por intermédio das alterações na constante dielétrica do meio. Essas alterações da capacitância do sensor forneciam informações, inclusive, para distinguir os tipos de materiais presentes no interior da parede. O princípio de funcionamento é apresentado na Figura 2.

Figura 2. Placas Capacitivas utilizadas na detecção de vigas no interior de paredes. [5]

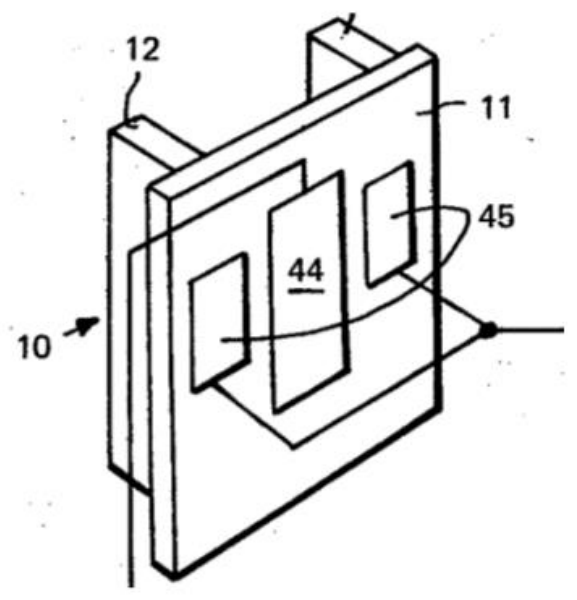

Fonte: R. C. Franklin. 
Conforme exibido na Figura 2 o sensor consistia em duas placas dispostas lateralmente, formando um capacitor e tendo como dielétrico a parede próxima ao dispositivo. [5]

\subsubsection{Radiofrequência}

As tecnologias baseadas em radiofrequência surgiram como uma solução mais robusta em relação aos métodos antes utilizados para detecção de objetos embutidos. Tecnologias de radiofrequência são baseadas nas técnicas de reflectometria, com emissão de ondas eletromagnéticas de alta frequência que são refletidas na fronteira entre dois meios de permissividades distintas. Assim como na operação dos radares, o tempo decorrido entre o sinal transmitido e recebido é utilizado para inferir a distância entre o sensor e a superfície refletora.

Figura 3. Sinal de radiofrequência com reflexões. [6]

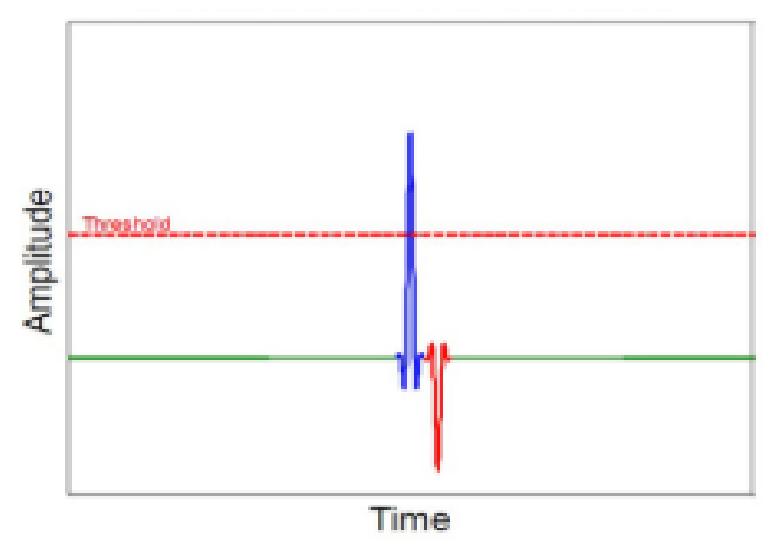

Fonte: Electronic Design.

Os radares que operam no ar e no espaço possuem um alcance que pode chegar a milhares de quilômetros. Entretanto, uma vez que o objetivo do projeto é detectar materiais através de uma superfície, é possível identificar mais correlação com radares de penetração no solo (Ground Penetrating Radar - GPR). Estes radares GPR possuem um alcance máximo de 100 metros operando na faixa de frequência de $500 \mathrm{MHz}$ a 1,5Ghz. A avaliação dos equipamentos de mercado é suficiente para definir que trabalhar em altas frequências, embora prejudique o alcance máximo do radar, permite a penetração em materiais utilizados na construção civil, como cimento, tijolos e concreto. [7]

Os principais métodos para detectar objetos através da parede estão exibidos no Quadro 1, o qual também apresenta uma comparação entre estes. As tecnologias que serão destacadas são consideradas as mais eficientes para a detecção de desvios e são possíveis tecnologias para serem utilizadas no princípio do STW (See Through Wall) que é caracterizado pela emissão de ondas eletromagnéticas capazes de penetrar em estruturas com a finalidade de realizar o mapeamento de objetos ou pessoas. 


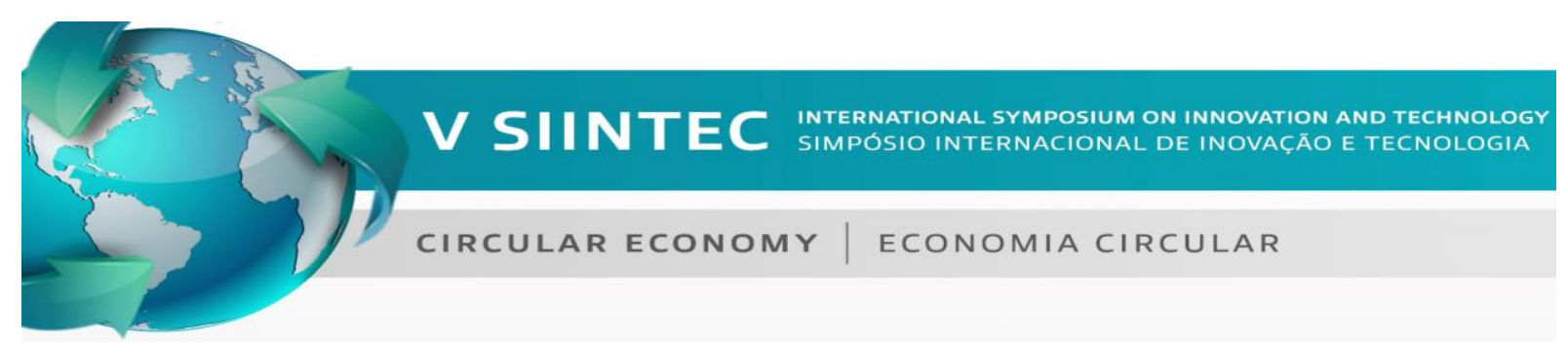

Quadro 1. Comparação entre as tecnologias eletromagnéticas.

\begin{tabular}{|c|c|c|}
\hline Técnicas & Vantagens & Desvantagens \\
\hline Microondas & $\begin{array}{l}\text { Boa Condução; } \\
\text { Transmite grande quantidade de } \\
\text { dados. }\end{array}$ & Necessidade de repetidores. \\
\hline Ultrassom & $\begin{array}{l}\text { Medição através de chapas de } \\
\text { metal; } \\
\text { Emissões direcionais; } \\
\text { Sensibilidade a pequenas } \\
\text { descontinuidades. }\end{array}$ & $\begin{array}{c}\text { Resolução Baixa; } \\
\text { Afetados pelo material do objeto e o } \\
\text { ângulo de incidência da onda; } \\
\text { A velocidade pode sofrer alterações pela } \\
\text { mudança nas propriedades do material. }\end{array}$ \\
\hline Radar convencional & $\begin{array}{l}\text { Penetração em nuvens, fumaça } \\
\text { e chuvas; } \\
\text { Não dependem de iluminação; }\end{array}$ & $\begin{array}{c}\text { Apresenta baixa resolução espacial; } \\
\text { Poucas informações acerca do objeto } \\
\text { detectado são fornecidas; } \\
\text { Baixo desempenho ao detectar pequenos } \\
\text { objetos; } \\
\text { Alto custo. }\end{array}$ \\
\hline $\begin{array}{l}\text { Imagem Térmica } \\
\text { (Termografia) }\end{array}$ & Velocidade de inspeção. & $\begin{array}{c}\text { Baixa penetração; } \\
\text { Pequenas discrepâncias mascaradas, } \\
\text { pelo "ruído de fundo"; } \\
\text { Os elementos inspecionados devem ser } \\
\text { relativamente finos; } \\
\text { A variação da corrente do elemento sob } \\
\text { inspeção pode ocasionar variações na } \\
\text { medição; }\end{array}$ \\
\hline Detecção Magnética & $\begin{array}{l}\text { Inspeciona defeitos cobertos ou } \\
\text { ocultos por obstáculos. }\end{array}$ & $\begin{array}{l}\text { Pouca precisão e sem capacidade de } \\
\text { detectar materiais diversos. }\end{array}$ \\
\hline $\begin{array}{l}\text { Raio X } \\
\text { (X-Ray) }\end{array}$ & $\begin{array}{c}\text { Método não invasivo e eficaz } \\
\text { para a técnica de STW, além de } \\
\text { ser preciso. }\end{array}$ & $\begin{array}{l}\text { Tecnologia cara, pouco portátil e implica } \\
\text { em maior capacitação e possíveis } \\
\text { adicionais aos profissionais. }\end{array}$ \\
\hline Radar UWB & $\begin{array}{l}\text { Tecnologia precisa e explorada } \\
\text { para STW; } \\
\text { Ė portátil. }\end{array}$ & $\begin{array}{l}\text { Precisa que a parede não seja muito } \\
\text { espessa ou de material atenuante na } \\
\text { banda de frequência usada. }\end{array}$ \\
\hline Sensores Capacitivos & $\begin{array}{l}\text { Geração de imagens sem } \\
\text { varredura mecânica; } \\
\text { Sensível a defeitos } \\
\text { superficiais e ocultos em } \\
\text { materiais isolantels. }\end{array}$ & $\begin{array}{c}\text { Pouco preciso; } \\
\text { Não distingue materiais com } \\
\text { profundidades distintas. }\end{array}$ \\
\hline
\end{tabular}

Fonte: Própria, 2019.

O quadro 1 demonstra as tecnologias existentes que foram mapeadas, sendo o ponto de partida para seleção das viáveis para detecção dos objetos embutidos. Após avaliação da viabilidade técnica, a comparação dos métodos em relação à aplicação restringiu a gama de equipamentos a serem testados pelo projeto.

\subsubsection{Sensores Indutivos}

Uma outra classe de dispositivos que pode ser utilizada para detectar objetos metálicos são os sensores indutivos. Estes equipamentos possuem uma ótima precisão, porém, só são capazes de detectar objetos em pequenas distâncias. Eles são compostos por um núcleo de ferrite envolto por uma bobina, um circuito oscilador e, por fim, um circuito disparador em conjunto com um amplificador (Disparador de Schmitt), como apresentado na Figura 4. 
Figura 4. Esquema de funcionamento do sensor indutivo. [8]

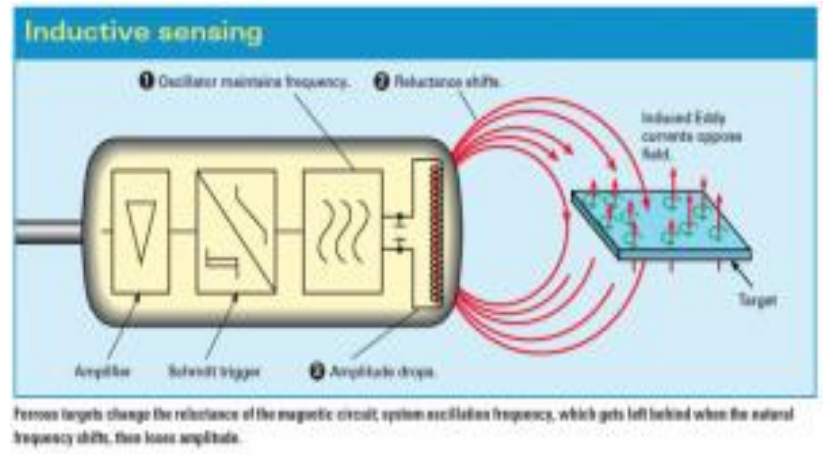

Fonte: Citisystems

O princípio de funcionamento do sensor indutivo se dá a partir de um campo eletromagnético variável que é gerado pelo oscilador em conjunto com a bobina na extremidade do dispositivo. Quando um material metálico penetra este campo, são induzidas pequenas correntes parasitas. Com a indução no metal, ocorre uma diminuição na energia do campo e, consequentemente na amplitude do sinal proveniente do oscilador. Quando este sinal se torna muito baixo, o circuito de disparo percebe a mudança e altera a tensão de saída. Fornecendo uma resposta lógica, de nível alto ou baixo, que pode ser utilizada no controle do processo. Assim, os sensores indutivos podem ser aplicados em diferentes funções relacionadas à percepção de proximidade. [8]

\subsubsection{Sensores de Efeito Hall}

Os sensores de Efeito Hall são caracterizados como um método não invasivo para detecção de corrente de maneira indireta, pois, através desses, é possível detectar a magnitude de campos magnéticos e, a partir de então, determinar valores de corrente. Estes campos são detectáveis por sua força ou interação em cargas elétricas, ímãs e produtos magnéticos. Estes dispositivos são transdutores que atuam com base na diferença de potencial gerada em um objeto condutor quando submetido a um campo magnético perpendicular ao fluxo de corrente que passa sobre ele, como mostrado na Figura 5.

Figura 5. Semicondutor Sob Ação de Campo Magnético. [9]

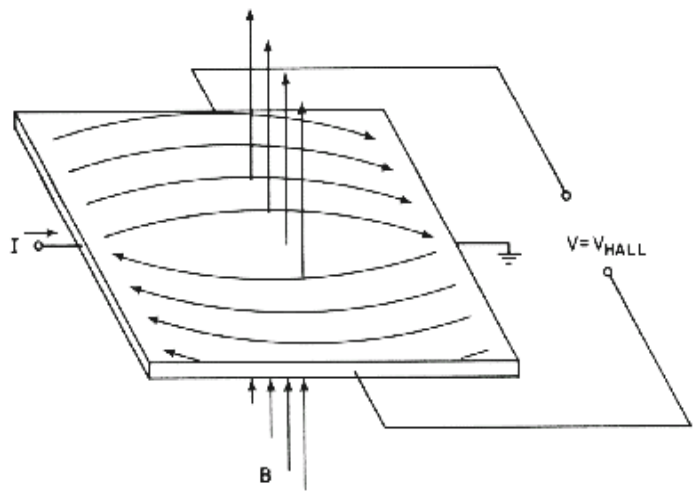

Fonte: UFPR, 2000. 
Os sensores de efeito hall podem se revelar alternativas úteis a serem validadas para a detecção de corrente, durante o processo de inspeção das concessionárias para localização dos desvios embutidos. Tais componentes serão testados nas fases posteriores do projeto.

\section{CONCLUSÃO}

O presente artigo abordou as principais técnicas eletromagnéticas que podem ser aplicadas para detectar fraudes embutidas em paredes. Dentre estas, destacamse os sinais de radiofrequência, os sensores indutivos e os sensores de efeito hall, os quais podem ser úteis para verificações e comprovações quanto ao ilícito.

Baseado nos diferentes pontos positivos e negativos apresentados pelas técnicas, pode-se destacar os métodos que manipulam os sinais de maneira que sejam capazes de penetrar as paredes, mapeando os objetos que se encontram em seu interior. Recurso que possibilitará o desenvolvimento de um protótipo capaz de detectar as fraudes embutidas a partir do imageamento e da análise dos valores de corrente, gerando provas do furto de energia de maneira rápida, eficiente e assertiva.

As características identificadas nas tecnologias levantadas se mostram apropriadas para as concessionárias de energia elétrica, tornando a ação de combate às perdas mais ágil e barato. Com isto, se espera a redução dos prejuízos decorrentes do furto e eliminação de constrangimentos ao consumidor, com a adoção de técnicas menos invasivas de inspeção.

\section{Agradecimentos}

À Light Serviços de Eletricidade S/A pelo financiamento do projeto de pesquisa e desenvolvimento do protótipo, vinculado ao Programa de P\&D do Setor Elétrico da Agência Nacional de Energia Elétrica (ANEEL). 


\section{REFERÊNCIAS}

${ }^{1}$ ANEEL. Resolução ANEEL no 338 de 25 de agosto de 2008. Disponível em: <http://www2.aneel.gov.br/cedoc/ren2008338.pdf>. Acesso em: 23 jul. 2008.

${ }^{2}$ ANEEL. Perdas de Energia. Disponível em: <http://www.aneel.gov.br/metodologiadistribuicao/-/asset_publisher/e2INtBH4EC4e/content/perdas/654800?inheritRedirect =false $>$. Acesso em: 23 jul. 2019.

${ }^{3}$ CPFL. CHAMADA PÚBLICA PD\&I N 001/2018 - Tema: Perdas não técnicas. Disponível em: <https://www.cpfl.com.br/energias-sustentaveis/inovacao/chamadapublica/Documents/EDITAL_TEMA\%2001_PERDAS\%20N\%C3\%830\%20TECNICA S.pdf>. Acesso em: 22 jul. 2019.

${ }^{4}$ ANEEL. Revista de P \& D. Disponível em: <http://www.aneel.gov.br/revista-de-p-d.> Acesso em: 22 jul. 2019.

${ }^{5}$ FRANKLIN, R.C.; FULLER, F. I. Electronic wall stud sensor. Disponível em: $<$ https://patents.google.com/patent/US4099118A/en>. Acesso em: 23 jul. 2019.

${ }^{6}$ E. Design. What's The Difference Between Measuring Location By UWB, Wi-Fi, and Bluetooth?. 06 Fevereiro 2015. Disponível em: <https://www.electronicdesign.co $\mathrm{m} /$ communications/what-s-difference-between-measuring-location-uwb-wi-fi-and-blue tooth>. Acesso em: 23 jul. 2019.

7 FRADEN, Jacob. Handbook of Modern Sensors: Physics, Designs, and Applications. SpringerVerlag, 2008.

8 SILVEIRA, C. B.; LOPES, G. C. Sensor Indutivo: 0 que é e como funciona. Disponível em: <https://www.citisystems.com.br/sensor-indutivo/>. Acesso em: 14 ago. 2019.

${ }^{9}$ A. B. d. R. M. Cavalcanti. Uma Avaliação da interferência entre redes 802.11G. Disponível em: <https://www.ufpe.br/documents/39830/1359036/177_Arthu rCavalcanti/df04e707-e096-41e9-b58d-4ea6cda4985e>. Acesso em: 24 jul. 2019. 